\title{
Medical Image Segmentation based on PSO-PFC
}

\author{
Gundeep Bajwa \\ Student (M.Tech) CSE \\ GIMET, Amritsar \\ PTU Regional Centre
}

\author{
Harwant Singh Gill \\ Supervisor (M.Tech) CSE \\ Assistant Professor \\ GIMET, Amritsar
}

\begin{abstract}
The increasing influence of segmentation in medical image processing requires great need to develop robust image segmentation method to eliminate the problem of traditional methods. Fuzzy c-means (FCM) is an effective fuzzy clustering technique for medical image segmentation but FCM is noise-sensitive and time consuming with large set of medical images. A penalized fuzzy clustering (PFC) is implemented for eliminating noise sensitivity of FCM. This paper presents the hybrid approach that employs Particle swarm optimization (PSO) to optimize the results of PFC for medical images segmentation.
\end{abstract}

\section{General Terms}

Image processing, segmentation, medical images, clustering.

\section{Keywords}

Medical image segmentation, fuzzy c-means (FCM), penalized fuzzy clustering (PFC), particle swarm optimization (PSO).

\section{INTRODUCTION}

Medical image segmentation is the main applied domain in the image segmentation. Medical imaging is used to create images of human body for clinical purposes. Medical imaging is performed in various modalities such as MRI, CT, ultrasound etc [11]. Image segmentation plays a crucial role in various medical imaging applications by automating or facilitating the delineation of anatomical structures and other regions of interest. Automatic segmentation methods offer a mechanism for overcoming the tedium involved in the manual segmentation of large data sets, and also promise reproducibility which is difficult with manually-defined results [2]. However, significant problems must be overcome to achieve segmentation by automatic means and it remains an active research areas.

Automatic segmentation of medical images is difficult task as medical images are complex in natures. Medical images involve three main image related problems: overlapping grayscale intensities, uncertainity and in-homogeneity, partial volume effect. The methods of segmentation vary widely depending upon the imaging modality, specific application and other factors [2].

\subsection{Image segmentation methods}

Image segmentation is process of partitioning image into distinct regions by grouping together pixels with similar attributes such as grey level, color, contrast, brightness or texture etc [1]. Image segmentation methods can be categorized based on the following two properties of images:

- Detecting similarities. It means the partition of the images based on the abrupt changes in the intensity values. The methods of edge detection are based on this approach of segmentation.

- Detecting discontinuities. It means the partition of the images by grouping the similar regions based on the predefined criterion. The methods of thresholding are based on this approach of segmentation.

The basic methods of segmentation can be broadly classified as [11]: edge-detection, region-growing, thresholding and clustering. Thresholding is a simple, yet effective, way of partitioning an image into a foreground and background. This image segmentation technique is a type of image segmentation that isolates objects by converting gray scale images into binary images. Edge detection is based on identifying and locating sharp discontinuities in an image to form closed object boundaries. Compared to edge detection method, segmentation algorithms based on region are relatively simple. Region based methods, partition an image into regions that are similar according to a set of predefined criteria.

Clustering is an unsupervised learning task, where one needs to identify a finite set of classes known as clusters to classify pixels. A similarity criteria is defined between pixels, and then similar pixels are grouped together to form clusters. Clustering algorithms are classified as hard clustering, kmeans clustering, fuzzy clustering etc [6].

\subsection{Fuzzy clustering methods}

Many clustering strategies have been used for image segmentation, such as the hard clustering scheme and the fuzzy clustering scheme, each of which has its own special characteristics. The conventional hard clustering method restricts each point of the data set to exclusively just one cluster. K-means clustering technique is the example of hard clustering scheme [3].

The fuzzy clustering scheme has been evolved because of the drawbacks of the hard clustering methods. Hard clustering technique lack flexibility and ignore real life situations. Fuzzy clustering is based on the fuzzy set theory, which produced the idea of partial membership of belonging described by a membership function. In fuzzy clustering, data elements can belong to one or more cluster and associated with each element is a set of membership levels [5]. These membership levels indicate the strength of the association between that data element and a particular cluster. Fuzzy clustering is a process of assigning these membership levels and then using them to assign data elements to one or more clusters. Fuzzy cmeans (FCM), Possibilities c-means (PCM), Gaussian mixture decomposition (GMD), Fuzzy c-varieties (FCV) are some of the fuzzy clustering algorithms [6]. 


\section{MEDICAL IMAGE SEGMENTATION ALGORITHMS}

Medical imaging is the process of creating images of the human body (parts or functions) for clinical purposes such as diagnosis, examining disease, treatment planning or medical science. Medical imaging is performed in various modalities, such as MRI, CT, ultrasound, positron emission tomography (PET), etc [11]. The increasing number of medical imaging modalities and growing number of medical images requires the use of computers to facilitate image processing. Computer algorithms for the delineation of anatomical structures and other regions of interest are becoming increasingly important in assisting and automating medical image analysis and processing. These algorithms, called image segmentation algorithms, play a vital role in many medical-imaging applications, such as the quantification of tissue volumes, diagnosis, study of anatomical structure, treatment planning [2]. This paper is focusing primarily on the segmentation of MRI images [Fig 1].

\subsection{Segmentation by FCM}

Fuzzy c-means (FCM) is popularly used fuzzy clustering segmentation algorithm. FCM is a method of clustering which allows one piece of data to belong to two or more clusters. FCM clustering algorithm is one of the most commonly used unsupervised clustering technique in the field of medical imaging. This method was developed by Dunn in 1973 and improved by Bezdek in 1981 [6]. FCM approach computes the objective function value for image segmentation $\mathrm{s}$. The objective function of FCM uses the gray level pixel values intensity for image segmentation process. On the basis of the objective function and the clusters the centre values have been computed that helps for image segmentation [Fig 2].

Although the conventional FCM algorithm works well on most noise-free images, it has a serious limitation: it does not incorporate any information about spatial context, which cause it to be sensitive to noise and imaging artifacts [7]. To compensate for this drawback of FCM, a penalized fuzzy clustering (PFC) algorithm is proposed

\subsection{Segmentation by PFC}

In the process of image segmentation FCM does not include the spatial domain model for image segmentation so that is much sensitive to noise. To reduce the effect of the noise the objective function of the FCM algorithm has been converted so that it produces a threshold value for image segmentation process. That particular threshold value computed by the spatial information has been considered as the penalty factor for FCM thus approach is penalized fuzzy clustering (PFC) [Fig 3].

\subsection{Segmentation by PSO-PFC}

After the segmentation of the medical image using the PFC approach the Particle Swarm Optimization approach has been used for the process of optimization of the image coefficients on the basis of the fitness function. The PSO approach uses the different number of particles and iteration for computation of the parameters for image segmentation. PSO approach is initialized by using the group of random particles. Out of these particles the search has been done for best particle in the search space by generating generations. The fitness of each particle for every iteration has been evaluated by using the fitness function. $Z_{i}$ denotes the current position of the particle in the search space, $\mathrm{PB}_{\mathrm{i}}$ personnel best particle available in the single step of generations of the particle and $V_{i}$ denotes the current velocity of the particle [Fig 4].

In the processing of PSO approach the parameters have been initialized by using initial velocity and initial position for particles.

1. Initialization. In this step the particle of the image have been initialized using different initialization of particle by defining the dimension of search space, initial position and initial velocity.

2. Velocity Update. After every iteration the velocity of the particle has been updated on the basis of the distance of the particle from the best particle in the iteration. The best particles in the iteration have been computed by using fitness function. In this velocity updating process $\mathrm{C} 1$ and $\mathrm{C} 2$ constants values have been used as the variable velocity coefficients. W denotes the weight age defined.

3. Position updating after this process the positions of the particle have been get updated on the basis of the adjusted velocity.

4. Global Best after the process of position and velocity updating the particle best and global; best solution has been computed on the basis of the fitness value. The particle best is computed from the minimum the fitness from the single generation steps. Out of the particle best the minimum fitness function has been optimized as the global best particle.

5. Stopping Criteria after updating of each particles available in the swarm there is two types of criteria has been defined that are number of iterations and CPU time. On the basis of these two factors the optimization of the system has been stopped until the step 2 to 4 goes on repeating.

By computing the best particle from the swarms the best global particle has been computed for the image. On the basis of that global value the image has been segmented and hidden information has been extracted. After the segmentation process various parameters have been analyzed for performance evaluation of purposed system. 


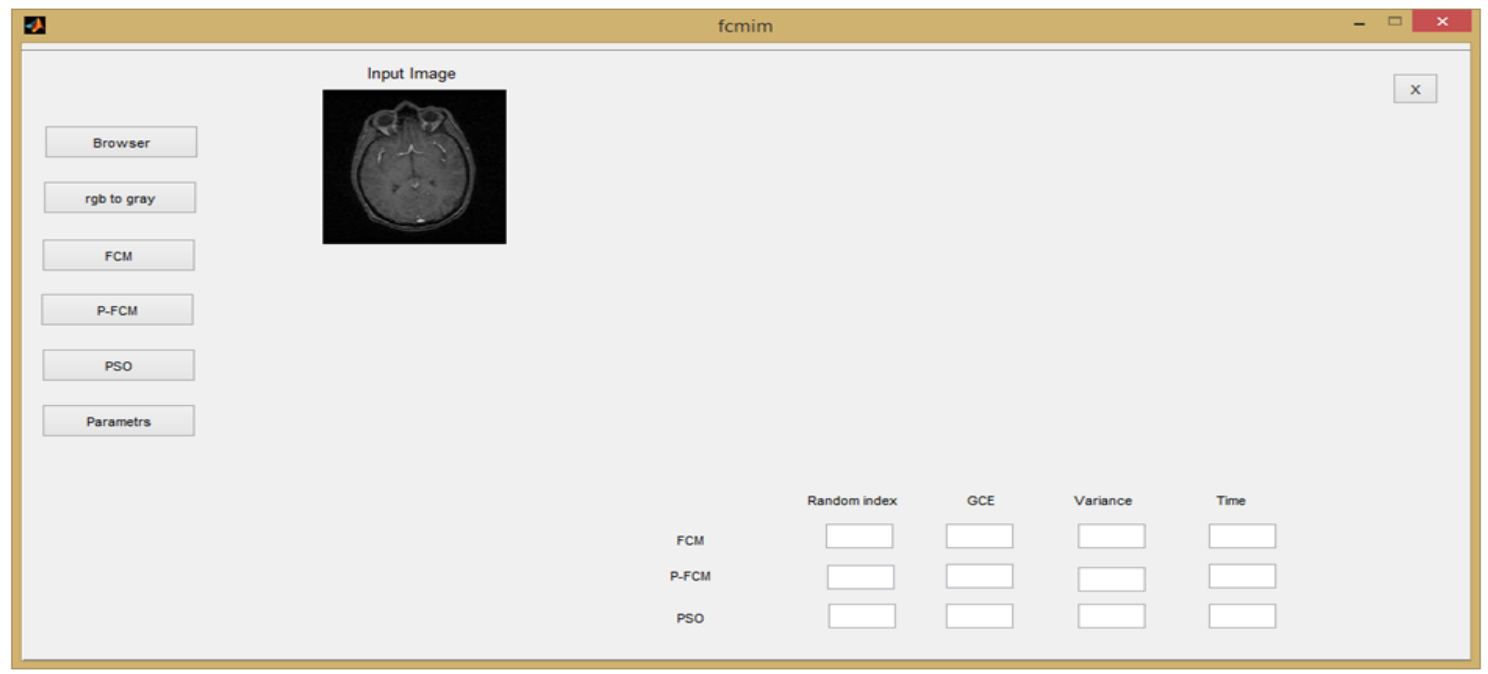

Fig1: Input Image for Image Segmentation

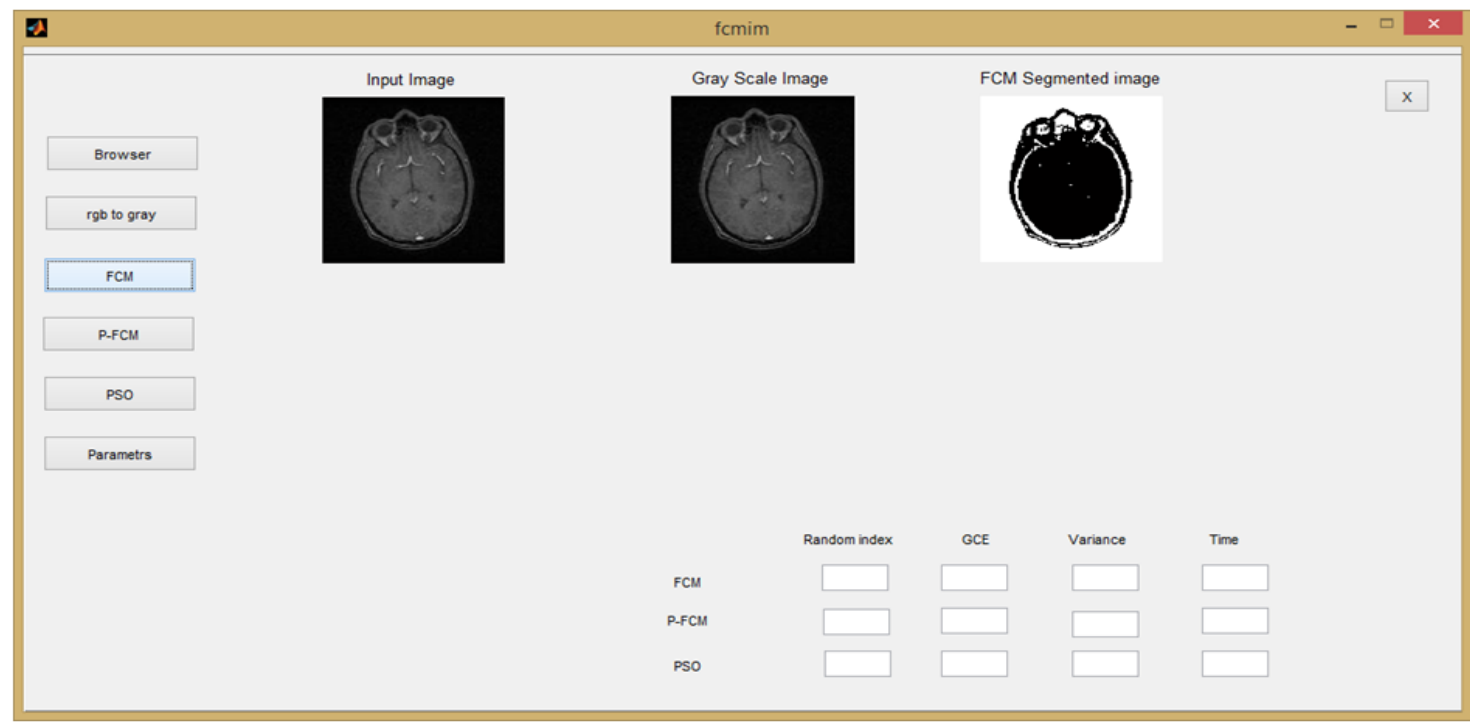

Fig 2: Image Segmentation using FCM approach

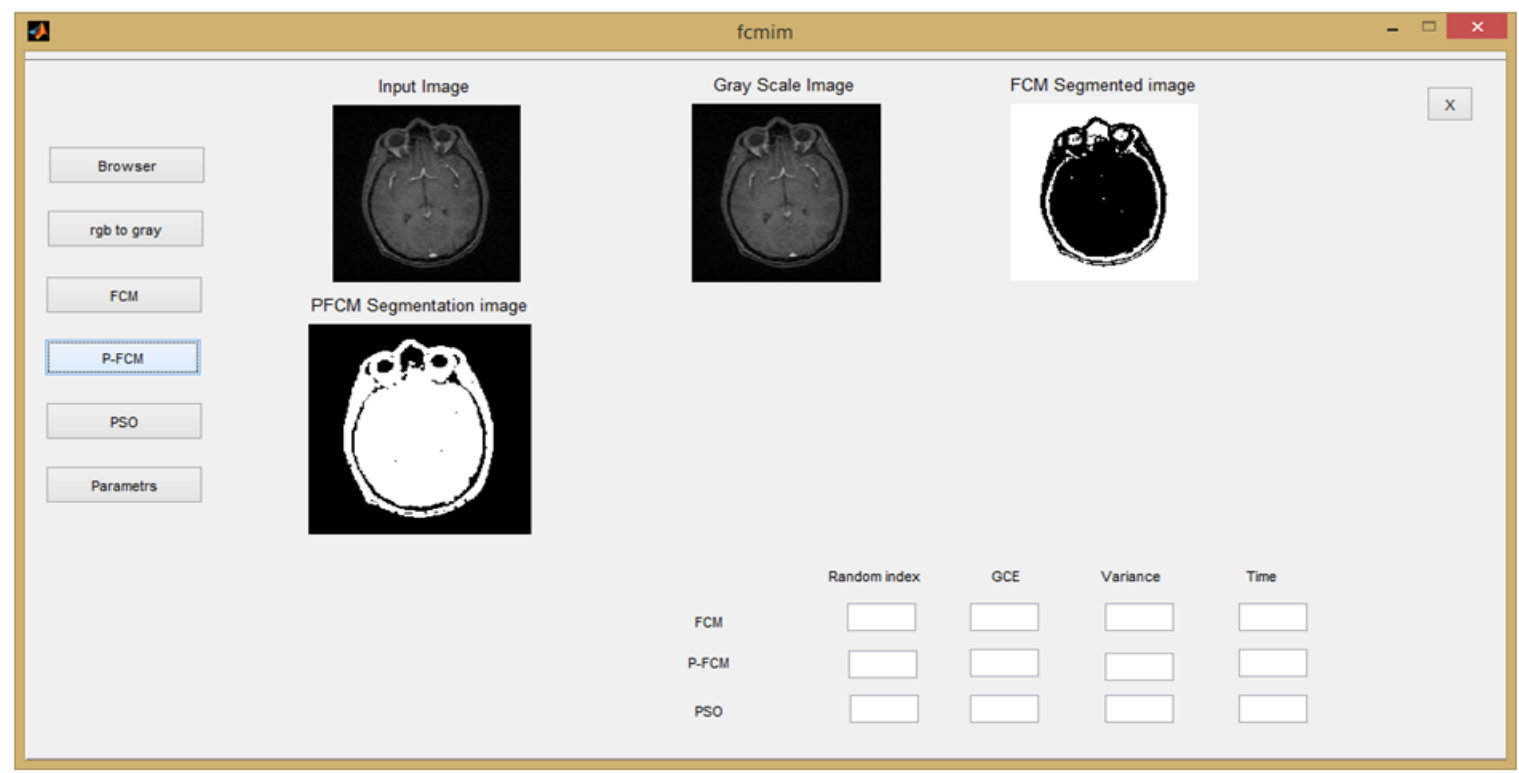

Fig 3: Image Segmentation using PFC approach 


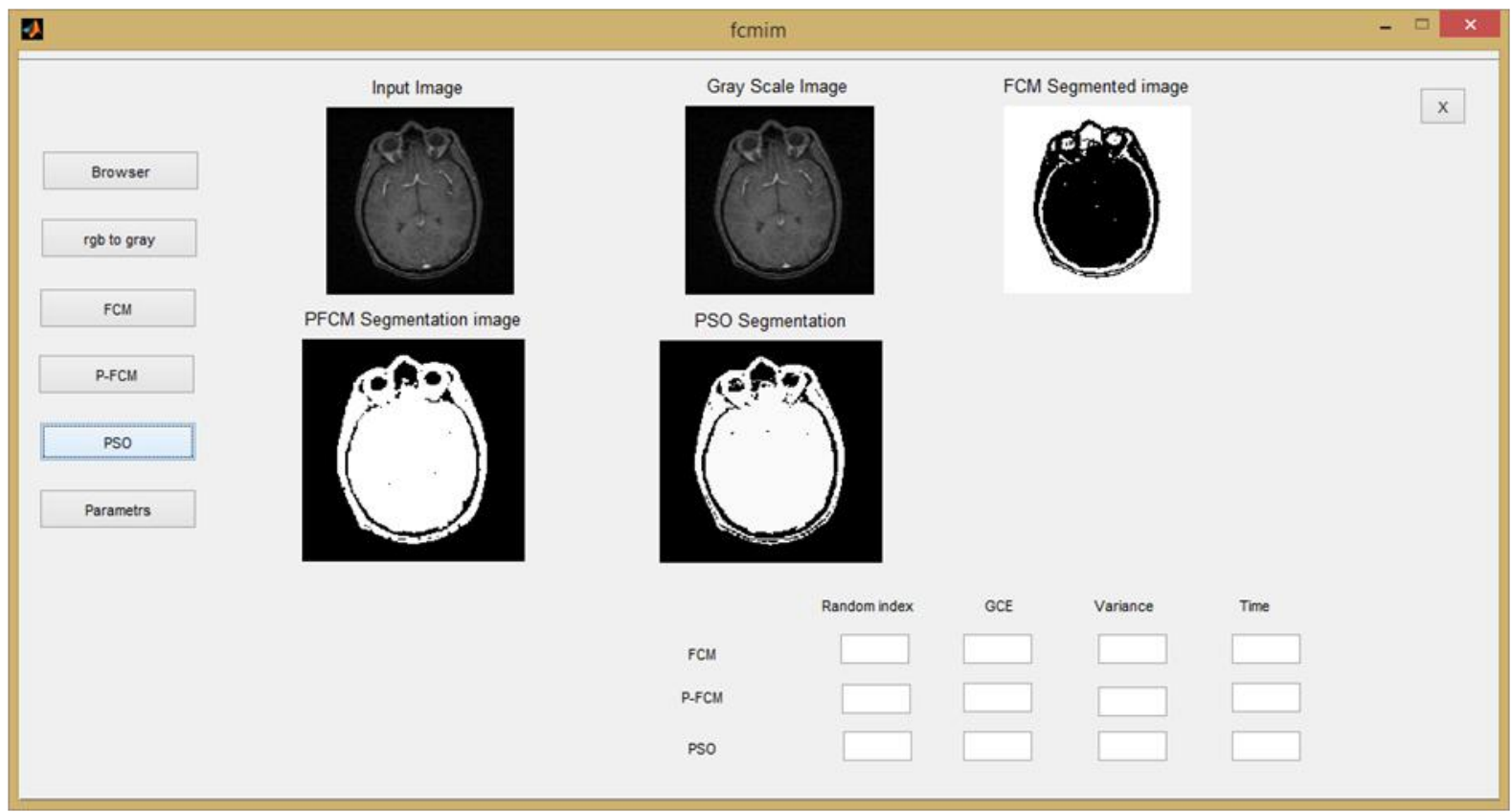

Fig 4: Optimization of PFC Segmentated Image using PSO

\section{RESULTS AND DISCUSSION}

In the purposed work different medical images has been used for image segmentation process. In particular, MRI medical images of different body parts have been used for image segmentation process. These are 300 images of different body parts have been available. These images are of heart, lungs, head, brain, and knee. The parameters random index, GCE, and variance have been computed for performance evaluation of purposed work.

\begin{tabular}{|c|c|c|c|}
\hline Images & FCM & PFC & PSO \\
\hline Heart & 1.42274 & 0.51884 & 0.509617 \\
\hline Brain & 1.4351 & 0.522 & 0.523096 \\
\hline Lungs & 1.4322 & 0.5168 & 0.5015 \\
\hline Knee & 1.422 & 0.537 & 0.501 \\
\hline
\end{tabular}

Table 1: Values of random index for different medical images

The table [1] presents the value of random index that has been computed for different medical images. Random index is the measure of the accuracy of image segmentation. The random index has a value between 0 and 1 , with 0 indicating that the two data clusters do not agree on any pair of points and 1 indicating that the data clusters are exactly the same.

\begin{tabular}{|c|c|c|c|}
\hline Images & FCM & PFC & PSO \\
\hline Heart & 0.678 & 0.033 & 0 \\
\hline Brain & 0.678 & 0.039 & 0 \\
\hline Lungs & 0.678 & 0.0026 & 0 \\
\hline Knee & 0.678 & 0.07033 & 0 \\
\hline
\end{tabular}

Table 2: Values of global consistency error for different medical images

The table [2] presents the values of global consistent error (GCE) for different medical images. GCE error measure is not symmetric and encodes a measure of refinement in one direction only.

\begin{tabular}{|c|c|c|c|}
\hline Images & FCM & PFC & PSO \\
\hline Heart & 6.07 & 4.85422 & 4.71601 \\
\hline Brain & 6.55 & 5.48 & 5.19552 \\
\hline Lungs & 5.77 & 4.59 & 4.41 \\
\hline & & & \\
Knee & 5.202 & 3.93 & 3.83 \\
\hline
\end{tabular}

Table 3: Values for variance for different medical images 
The table [3] presents the values of variance for different medical images which shows how far images are spread out. A small variance indicates that the data points tend to be very close to the mean (expected value) and hence to each other, while a high variance indicates that the data points are very spread out around the mean and from each other.

\section{CONCLUSION}

Image segmentation is the process for extraction the meaningful information from the images. In the process of image segmentation process in the process of the image segmentation various types of image have been used. In the purposed work MRI medical images have been used for image segmentation process. Fuzzy c-means (FCM) is an effective fuzzy clustering technique for medical image segmentation but FCM is noise-sensitive and time consuming with large set of medical images. A penalized fuzzy clustering (PFC) is implemented for eliminating noise sensitivity of FCM. After using the PFC approach the image coefficients have been optimized by using Particle Swarm Optimization Approach. PSO use the fitness function fo0r computation of globally best particles from the image. The various parameters that are random index, GCE and variance has been computed for both approaches. On the basis of these parameters we can conclude that PFC with PSO provides better results than other FCM approach.

\section{ACKNOWLEDGEMENT}

This paper is made with the support of parents and family members. I want to thanks them for their support, help and guide Special thanks to God for the power and guidance during the completion of my work.

\section{REFERENCES}

[1] H.P Narkhede, "Review of image segmentation techniques", IJISME, ISSN: 2319-6386, volume-1, issue8, July 2013.

[2] Lalit M. Aggarwal, Neeraj Sharma, "Automated medical image segmentation techniques", Journal of Medical Physics (J Med Phys.), 2010 Jan-March.
[3] Mahesh Yamba, Hitesh Gupta, "Image segmentation using fuzzy c-means clustering: A survey", IJARCCE, volume-2, issue-7, July 2013.

[4] Neelofar Sohi, Lakhwinder kaur, Savita Gupta, "Performance Improvement of Fuzzy C-mean Algorithm for Tumour Extraction in MR Brain Images", International Journal of Computer Applications (0975 8887), Volume 59- No.5, December 2012.

[5] Piyush Valvi, Brijesh Shah, Satish Shah, "Improved fuzzy c-mean algorithm for Medical Image segmentation”, IJERT, Vol. 1 Issue 3, May - 2012.

[6] R. Ravindraiah, K. Tejaswini, "A Survey of Image Segmentation Algorithms Based On Fuzzy Clustering", IJCSMC, Vol. 2, Issue. 7, July 2013.

[7] R.Suganya, R.Shanthi, "Fuzzy C- Means Algorithm- A Review", International Journal of Scientific and Research Publications, Volume 2, Issue 11, November 2012.

[8] Rajaei, Lalitha Rangarajan, Elham Dallalzadeh, "Medical Image Texture Segmentation", IJCSMC, Vol. 1, Issue 2, 2010.

[9] Soumi Ghosh, Sanjay Kumar Dubey, "Comparative Analysis of K-Means and Fuzzy C-Means Algorithms", International Journal of Advanced Computer Science and Applications (IJACSA), Vol. 4, 2013.

[10] Rajeev V, Dr Sreeja Mole, "FCM Algorithm for Medical Image Segmentation using HMRF", IJESRT, September 2013.

[11] Sonal G. Deore, Dinesh D. Patil, "Medical Image Segmentation: A Review", IJCSMC, Vol. 2, Issue. 1, January 2013.

[12] Yang Zhang, Chung Fu-Lai, "Robust fuzzy clustering based image segmentation", Applied soft computing, volume 2, no. 1, January 2002. 